\title{
A SYNERGISTIC EFFECT IN THE MEASUREMENT OF NEURO-FUZZY SYSTEM
}

\author{
Sergey Gorbachev ${ }^{\mathrm{a}}$, Nadezhda Gorbacheva, Sergey Koynov \\ National research Tomsk state University, 634050, Tomsk, Russia
}

\begin{abstract}
We consider a new type of hybrid neuro-fuzzy system based on fuzzy and neural computing in hierarchical sequential structure, the total effect exceeds the effect of each component separately. The proposed system can be applied to multi-criteria analysis, automatic classification on signs and obtain evidence-based estimates of the efficiency of scientific and technical solutions and technologies, engineering and robotics. An example of a neuro-fuzzy system measuring the intensity of the emotions of a robot, with the extraction of diagnostic decision rules "If ... then".
\end{abstract}

\section{Introduction}

The concept of soft computing (soft computing) was first mentioned in the work of Zadeh (Lotfi A. Zadeh) in 1994 [1]. Soft computing - is a complex computer methodology based on fuzzy logic, evolutionary computation, Neurocomputing and probabilistic calculations, with the later inclusion of chaotic systems, trust networks and learning theory sections. Components do not compete, but they create a synergistic effect. The guiding principle of soft computing - is accounting inaccuracies, uncertainty, partial truth, and approximation to achieve robustness, low cost solutions that better fit with reality. Four constituents of soft computing include:

- Fuzzy logic - approximate calculations, granulation information on the calculation of the words.

- Neurocomputing - learning, adaptation, classification, system modeling and identification.

- Evolutionary computation - synthesis, configuration and optimization using systematic random search and evolution.

- Probability calculations - uncertainty management, trust networks, chaotic systems, prediction.

Traditional computer calculations (hard computing) and the measurement is too precise for the real world. There are two classes of problems for soft computing and measurement: First, there are problems, for which complete information can not be obtained, and, secondly, the problem is not defined adequately.

Each individual component of soft computing has a number of internal problems, mutual compensation and which creates a synergistic effect. Neural networks solve the problem of approximation or classification, and setting the balance is due to the training.

\footnotetext{
${ }^{a}$ Corresponding author: gsv@mail.tsu.ru
} 
But the knowledge distributed across many affairs and results of the neural network seem inexplicable to the user, that is needed explication of knowledge. Fuzzy inference systems, on the contrary, have explicit knowledge in the form of products and make it easy to build explanations protocol. However, this clarity is achieved by a long and creative in nature prior knowledge extraction process and subsequent debugging set of rules. The inability to extract knowledge from data, settings membership functions during training, automatic reduction of the rules - the problem of inference systems. Application of genetic algorithms requires a prior study of the problem in order to select the model of probability, the formulation of the objective function. Selection soft computing individual components as a base typically defines the overall system architecture of a hybrid (a hybrid system in this case it refers to the "soft" system).

In [2] it is noted that in dealing with classification tasks to measure the effectiveness of multidimensional objects in recent years there has been an increase in the number of socalled hybrid algorithms, in which two or more soft computing technology integrated (fuzzy logic, neural networks, genetic algorithms) to improve the overall performance algorithms. Fuzzy Neural Network - a fuzzy inference system by the methods of neural networks. The conclusion is based on the apparatus of fuzzy logic, but the parameters of membership functions are configured using neural networks learning algorithms. Therefore, for the selection of the parameters of such networks use the method of back propagation, initially proposed for training of multilayer perceptron. For this fuzzy control module is in the form of the multilayer network, consisting usually of four layers: the layer of input variables fuzzyfication, the layer of activation conditions aggregation, the layer of fuzzy rules aggregation and the output layer (Figure 1).

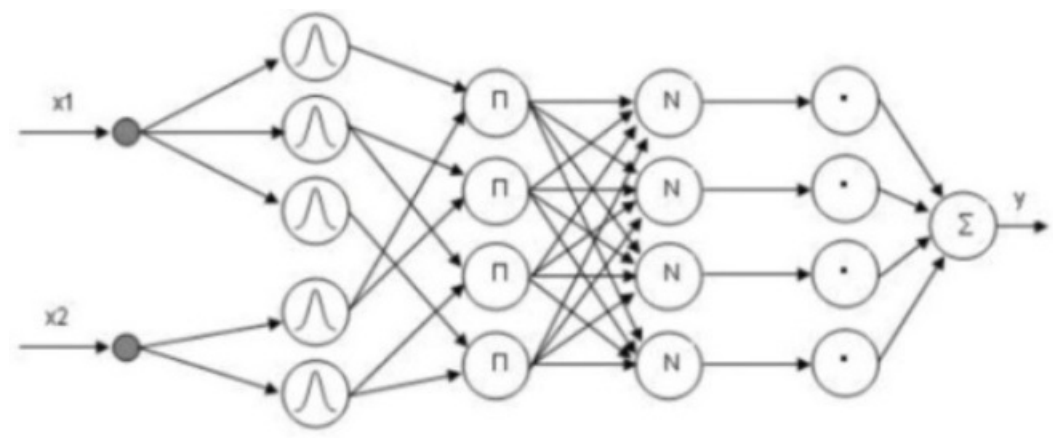

Figure 1. An example of a hybrid neuro-fuzzy.

Currently classifiers built using fuzzy neural networks, expert systems, and other methods, allow to obtain sufficiently accurate results, however, lead to the construction parametrically complicated, cumbersome models with a large number of neurons, and, consequently, to increase the amount of memory and time the network. The analysis of the literature reveals a contradiction, which is the lack of complete solutions for the construction of simple and compact classifier providing a compromise between accuracy and interpretability of classification rules.

\section{Purpose and Method}

The purpose of this research is to develop algorithms for constructing a hybrid neuro-fuzzy classifier for analyzing and measuring the intensity of the emotion of the robot, 
characterized by a set of disparate parameters, which provides not only a high classification accuracy, but also consists of a compact and interpreted a set of classification rules.

\section{Results and discussion}

In the domestic and foreign literature describes the use of decision trees as a powerful evolutionary methodology for solving problems of classification and regression [3]. The following simple example (on the IBM SPSS Modeler Infocenter Site) shows a decision tree for making a car purchase (Figure 2).

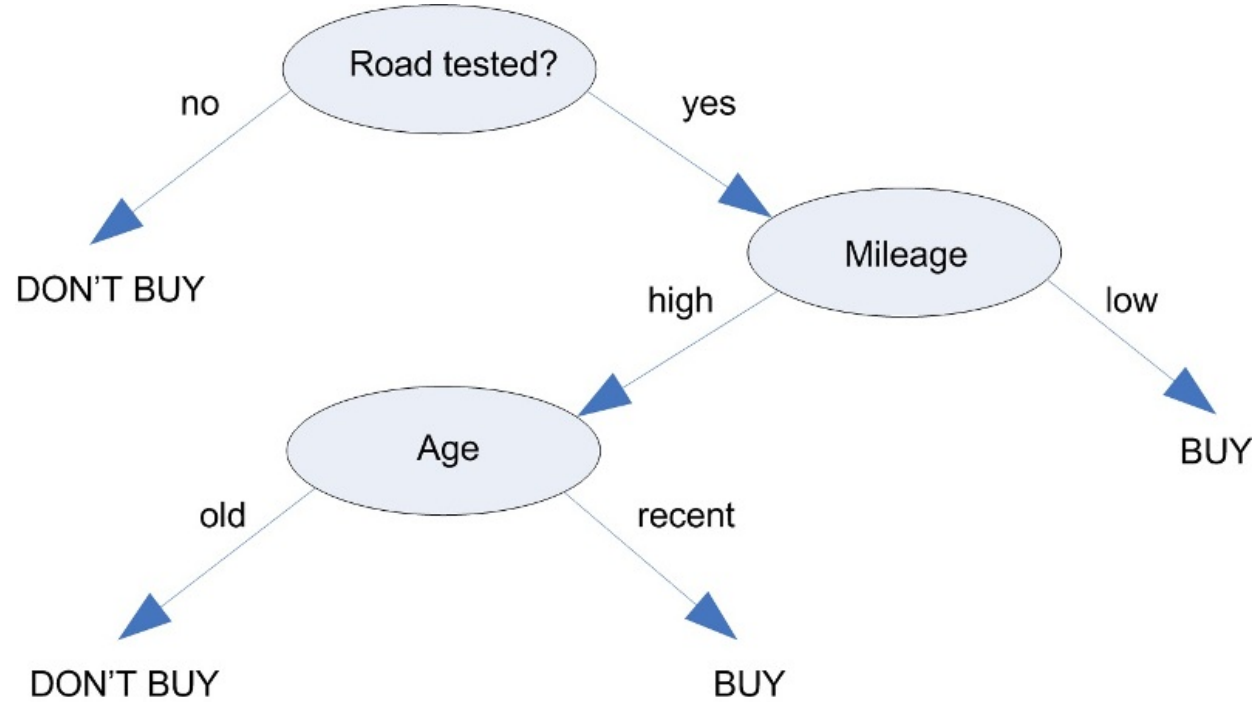

Figure 2. A decision tree for making a car purchase.

In Figure 2 shows the classification tree iris. Classification is into three classes and passes in the parameters: length \thickness of the sepal (SepalLen, SepalWid) and length \thickness of the petal (PetalLen, PetalWid). As you can see, each node should belong to the class (depending on which items got more in this node), the number got there of observations $\mathrm{N}$ and the number of each class. Also not the leafy tops, is the condition of the transition is one from a child. Respectively, for these conditions and breaks the sample. As a result, this tree almost perfectly (6 out of 150 incorrectly) klassificeret the original data on which it was trained.

If the monitoring condition is true, then the transition on the left edge, if false - on the right. Typically, each node includes examining one independent variable. Sometimes in the tree node, two independent variables are compared to each other or determined by some function of one or several variables.

If a variable that is checked at the node that takes categorical values, each possible value corresponds to a branch emerging from a node of the tree. If the value of the variable is a number, verified by more or less than this value a constant. Sometimes the area of numeric values is divided into intervals (the test value falls in an interval). The leaves of the trees correspond to the values of the dependent variable, i.e. classes.

A common disadvantage of the development of traditional decision trees is the requirement of certainty of the input data, which is achieved by applying weighted average values of input parameters that may result in significantly displaced resulting point estimates. It is also obvious that the requirement of determinacy of input data is an 
unjustified simplification of reality, as real complex objects of analysis are characterized by many uncertainties: uncertainty of initial data, the uncertainty of the external environment, the uncertainties associated with the nature, options, and model implementation or the object's state.

To improve the accuracy of classification and decision-making, the author developed the algorithm for constructing neuro-fuzzy decision tree having the property of adapting parameters using neural network learning. As input data we use a fuzzy set with the membership function of the following form (Figure 3).

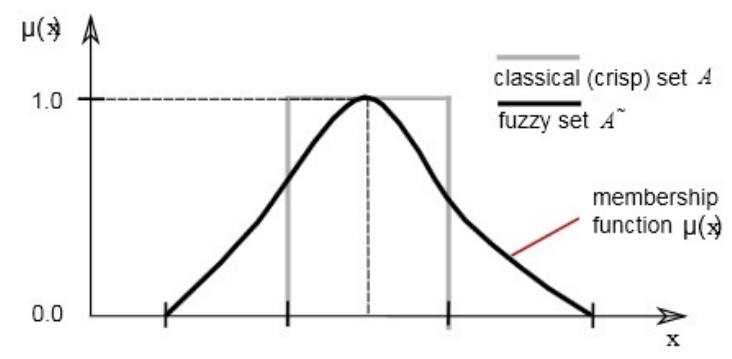

Figure 3. The membership function of fuzzy set $A$.

For any set $X$, a membership function on $X$ represent fuzzy subsets of $X$. The membership function which represents a fuzzy set $A^{\sim}$ is usually denoted by $\mu_{A}$. For an element $x$ of $X$ the value $\mu_{A}(x)$ is called the membership degree of $x$ in the fuzzy set $A^{\sim}$. The membership degree $\mu_{A}(x)$ quantifies the grade of membership of the element $x$ to the fuzzy set $A^{\sim}$. The value 0 means that $x$ is not a member of the fuzzy set; the value 1 means that $x$ is fully a member of the fuzzy set. The values between 0 and 1 characterize fuzzy members, which belong to the fuzzy set only partially.

In the forward loop fuzzy decision trees are built based on the fuzzy ID3 algorithm [3]. Algorithm The Iterative Dichotomizer 3" (ID3) is one of the earliest algorithms for learning decision trees (1985), using recursive partitioning subsets in the nodes of the tree on one of the selected attributes, and is widely used in problems of decision-making. In General it is as follows - starts with the root of the tree, which contains all examples of the training set. To separate it selects one of the attributes, and for each the values it accepts the construction branch, and you create a child node in which distributed its containing record.

The procedure is repeated recursively until then, until the knots will not be only examples of one class, then they will be declared the leaves and the branching stops. The most problematic step here is the choice of which attribute should be split. The classic ID3 algorithm uses a criterion increase information or decrease in entropy.

In the loop feedback parameters $[4,5]$ of fuzzy decision trees proposed to adapt based on the stochastic gradient algorithm by traversing back from leaf to root nodes. With this strategy in the course of adaptation parameters, the hierarchical structure of fuzzy decision trees intact, and the neural network back-propagation algorithm directly on the structure of fuzzy decision trees improves the accuracy of training without compromising interpretability.

Note that in order to reduce input information and presenting it in understandable to interpret the form of the algorithm was developed gazifikatsii input data based on the Kohonen self-organizing maps, the Value of fuzzy input parameters, described in linguistic terms, can be represented functionally by a set of membership functions. For the given set 
of functions, we find several new fuzzy sets, which are considered as the result of clustering the original data for describing a plurality of membership functions (Figure 4).

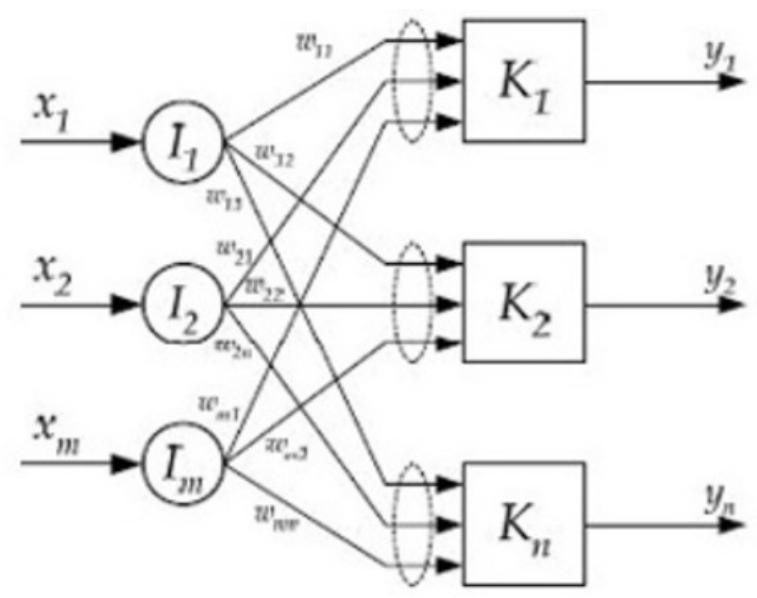

Figure 4. The algorithm fuzzy- input data by Kohonen self-organizing maps .

\section{Insights}

On the basis of the conducted research we can draw the following conclusions. To solve the problem of multidimensional measuring the intensity of the emotions generated by the emotional agent for the robot to vliyaniyem diverse input parameters, as a neuro-fuzzy system can use not the classical 4-layer network (Figure 1), and neuro-fuzzy decision tree structure which is constructed by the method of self-organization by fuzzy ID3 algorithm and for tuning of parameters (membership functions and identification of sheets) are used to reverse the cycles of iteration neural network method with back propagation mistakes. This hybrid neuro-fuzzy system based on a combination of methods of "sequential hierarchy" (decision trees) + fuzzy logic + neural networks". Hence, in weakly structured environments multi-criteria analysis of the appropriate joint use of these methods for modelling of emotional agent robot, is used to support decision-making in robotic systems, as a compensation for the frailties of the individual component of the methodology of soft computing expands the possibilities for building intelligent systems with incomplete input information. Such hybrid neuro-fuzzy system have the most powerful cognitive capacities (modelling sensation, perception, recognition, learning and remembering patterns with the purpose of revealing of knowledge from data). They find a substantially wider field of application than other methods of synthesis of fuzzy sets and neural networks. This is because such systems enable the best use of the "strong" side of a fuzzy system interpretability of knowledge) and neural networks (the ability to learn on the data).

\section{Conclusion}

In the course of the study of neuro-fuzzy systems were obtained the following results:

1. The developed method of synthesis of neuro-fuzzy classifier based on decision tree, neural network includes adapting parameters of the constructed decision tree. The model focuses on the input vector data does not depend on the mutual ratios of the elements of the 
input vectors does not use the learning procedure used in neuro-fuzzy models with the teacher, and eliminates the problem of choice of their structure.

2. Determined that the proposed neural network method for adapting parameters of a decision tree based on the backpropagation of the signal from the leaves of the tree to the root, in contrast to the known optimization problems, improves the classification accuracy of fuzzy decision trees a fuzzy system with direct distribution of signal) without prejudice to the interpretation, preserving the hierarchical structure of the tree.

3. In contrast to the known method of synthesis of neuro-fuzzy networks the training set the method of display the rules in its structure [6], leading to the construction of a parametrically complex, cumbersome model with a large number of neurons, and, consequently, to increase the amount of memory and uptime of the network, the method of synthesis of neuro-fuzzy classifier based on decision tree allows to synthesize models with a small number of elements with a high level of generalization, with the possibility of linguistic interpretation of the result by displaying diagnostic decision rules "If ... then".

In the General case described neuro-fuzzy system allows automated to solve the difficult problem of multi-criteria analysis and measurement of analysis objects having an arbitrary shape, with the intersection of classes is characterized by high accuracy and efficiency for solving problems of decision support under uncertainty, the low training time, the reliability against noise and distortion of information.

\section{Acknowledgments}

The paper was written as part of the research project No. 8.2.31.2015, carried out with the support of the Program "Research Foundation of Tomsk State University named after D.I. Mendeleev" in 2015 - 2016., grant RFBR No. 16-29-04388\16.

\section{References}

[1] L.A. Zadeh, Com. of the ACM 37, 77 (1994) doi: 10.1145/175247.175255

[2] S.V. Gorbachev, V.I. Syryamkin, Neuro-fuzzy techniques in intelligent systems of processing and analysis of multidimensional information (Tomsk state University, Tomsk, 2014)

[3] L. Breiman, J.H. Friedman, R.A. Olshen, C.J. Stone, Classification and regression trees (Wadsworth \& Brooks/Cole Advanced Books \& Software, Monterey, 1984)

[4] D.V. Shashev, S.V. Shidlovskiy, Optoelectronics, Instrumentation and Data Processing 51, 227 (2015) doi: 10.3103/S8756699015030036

[5] D.V. Shashev, S.V. Shidlovskiy, V.I. Syriamkin, A.V. Yurchenko, IOP Conference Series: Materials Science and Engineering 81, 012101 (2014) doi: 10.1088/1757$899 X / 81 / 1 / 012101$

[6] N. Yarushkina, Int. J. General Systems 30 , 71 (2001) 